\title{
Fatty acid elongase 5 (ELOVL5) alters the synthesis of long-chain unsaturated fatty acids in goat mammary epithelial cells
}

\author{
H. B. Shi, ${ }^{\dagger} \dagger^{1}$ Y. Du, ${ }^{*}$ C. H. Zhang, $\ddagger$ C. Sun, ${ }^{*}$ Y. L. He, ${ }^{*}$ Y. H. Wu, ${ }^{*}$ J. X. Liu, † J. Luo, $\ddagger$ and J. J. Loor $\S^{1}$ \\ *Zhejiang Provincial Key Laboratory of Silkworm Bioreactor and Biomedicine, College of Life Sciences, Zhejiang Sci-Tech University, Hangzhou, \\ 310018, P. R. China \\ †College of Animal Sciences, Zhejiang University, Hangzhou 310058, P. R. China \\ $\ddagger$ College of Animal Science and Technology, Northwest A\&F University, Yangling, Shaanxi, 712100, P. R. China \\ §Mammalian NutriPhysioGenomics, Department of Animal Sciences and Division of Nutritional Sciences, University of Illinois, Urbana 61801
}

\begin{abstract}
Increased production of long-chain unsaturated fatty acids (LCUFA) can have a positive effect on the nutritional value of ruminant milk for human consumption. In nonruminant species, fatty acid elongase 5 (ELOVL5) is a key enzyme for endogenous synthesis of long-chain unsaturated fatty acids. However, whether ELOVL5 protein plays a role (if any) in ruminant mammary tissue remains unclear. In the present study, we assessed the mRNA abundance of ELOVL5 at 3 stages of lactation in goat mammary tissue. Results revealed that ELOVL5 had the lowest expression at peak lactation compared with the nonlactating and late-lactating periods. The ELOVL5 was overexpressed or knocked down to assess its role in goat mammary epithelial cells. Results revealed that ELOVL5 overexpression increased the expression of perilipin2 (PLIN2) and decreased diacylglycerolacyltransferase 2 (DGAT2) and fatty acid desaturase 2 (FADS2) mRNA, but had no effect on the expression of DGAT1, FADS1, and stearoyl-CoA desaturase 1 (SCD1). Overexpression of ELOVL5 decreased the concentration of C16:1n-7, whereas no significant change in $\mathrm{C} 18: 1 \mathrm{n}-7$ and $\mathrm{C} 18$ : 1n-9 was observed. Knockdown of ELOVL5 decreased the expression of PLIN2 but had no effect on DGAT1, DGAT2, FADS1, FADS2, and SCD1 mRNA expression. Knockdown of ELOVL5 increased the concentration of C16:1n-7 and decreased that of C18:1n-7. The alterations of expression of genes related to lipid metabolism after overexpression or knockdown of ELOVL5 suggested a negative feedback regulation by the products of ELOVL5 activation. However, the content of triacylglycerol was not altered by knockdown or overexpression of ELOVL5 in goat mammary epithelial cells, which
\end{abstract}

\footnotetext{
Received October 28, 2017.

Accepted December 29, 2017 edu

${ }^{1}$ Corresponding authors: shihengbo@zstu.edu.cn and jloor@illinois.
}

might have been due to the insufficient availability of substrate in vitro. Collectively, these are the first in vitro results highlighting an important role of ELOVL5 in the elongation of 16-carbon to 18-carbon unsaturated fatty acids in ruminant mammary cells.

Key words: elongase, unsaturated fatty acid, ruminant, milk fat

\section{INTRODUCTION}

Fatty acids are one of the most valuable components in dairy production (Shingfield et al., 2008), especially the MUFA. These MUFA are substrates for the synthesis of PUFA and, thus, contribute to human health (Han et al., 2017). The activity of fatty acid desaturases and elongases determines the synthesis of end products of MUFA or PUFA synthesis (Lee et al., 2016). In ruminant mammary tissue, the functional roles of fatty acid desaturases [e.g., stearoyl-CoA desaturase 1 (SCD1)] have been investigated (Cecchinato et al., 2012; Lee et al., 2016; Yao et al., 2017).

The fatty acid elongase or elongation of very long chain fatty acid-like fatty acid elongase (ELOVL) includes 7 isotypes (i.e., ELOVL1 to ELOVL7). The RNA sequence revealed that at least 3 isoforms of fatty acid elongase are expressed in goat mammary gland tissue (Shi et al., 2015a). The isoforms are ELOVL5, ELOVL6, and ELOVL\%. Recent data in goat mammary cells indicated that ELOVL6 plays a role in the elongation of long-chain SFA (C16:0 to C18:0; Shi et al., 2017a). The ELOVL5 is involved in the elongation of various PUFA containing 18 and 20 carbons (Green et al., 2010). These data indicated that ELOVL family may play an important role in mammary fatty acid metabolism. However, the role of ELOVL in the ruminant mammary gland remains unclear.

At least in nonruminants, the ELOVL5 protein is sensitive to diet and hormones (Zhang et al., 2016). In mice, elevating Elovl5 activity increased hepatic and plasma levels of dihomo- $\gamma$-linolenic acid (C20:3n 
-6), decreased hepatic arachidonic acid (C20:4n-6) and docosahexaenoic acid (C22:6n-3) content (Wang et al., 2008), and reduced hepatic triacylglycerol (TAG; Tripathy et al., 2014). In rat insulinoma-1 cells, knockdown of Elovl5 decreased elongation of C16:1n-7, whereas Elovl-5 overexpression increased synthesis of 18:1n-7 (Green et al., 2010). It is evident that ELOVL5 changed the hepatic fatty acid content through affecting multiple pathways related to lipid metabolism [e.g., sterol-regulatory element binding protein-1 (SREBP-1) signaling pathway (Wang et al., 2008; Moon et al., 2009) or Akt pathway (Tripathy et al., 2010)]. Collectively, the data from rodents underscore an important role for Elovl5 in the overall process of long-chain unsaturated fatty acid (LCUFA) synthesis.

Despite evidence in mice supporting an essential role of Elovl5 in PUFA synthesis, no functional data are available on ELOVL5 in ruminant mammary cells. Whether ELOVL5 plays a role in the synthesis and alteration of LCUFA composition in ruminant mammary cells remains unknown. We hypothesized that ELOVL5 has a role in the process of LCUFA synthesis in ruminant mammary cells. To assess the hypothesis, both RNA interference and overexpression were performed in goat mammary epithelial cells (GMEC).

\section{MATERIALS AND METHODS}

\section{mRNA Abundance of ELOVL5}

The transcriptome data set from goat mammary tissue is deposited at the National Center for Biotechnology Information (BioProject ID: PRJNA243005). The details about these samples were described previously (Shi et al., 2015a). Briefly, 3- to 4-yr-old Xinong Saanen dairy goats from the experimental farm of Northwest Agricultural University, Shaanxi, China, were used. Mammary tissue at peak lactation (3 goats, 100 d postpartum), late lactation (cessation of milking, 3 goats, $310 \mathrm{~d}$ postpartum), and the nonlactating period (3 goats, nonlactation and nonpregnant period) were collected by a veterinarian after slaughter. Upon RNA sequencing, Bowtie 0.12.8 was used to align reads to the goat genome (Dong et al., 2013). The DESeq (a software for differential gene expression analysis based on negative binomial distribution of data; http://www .huber.embl.de/users/anders/DESeq) was used for analysis of gene expression in response to stage of lactation. The single-end mapping method was used for read analysis; one read was compared with multiple genes. Reads per kilobase of exon model per million mapped reads were used for measuring gene expression of ELOVL5.

\section{Adenovirus Generation}

The whole process for generation and proliferation of recombinant adenovirus expressing ELOVL5 (AdELOVL5) was carried out as previously described (Shi et al., 2016). Briefly, the ELOVL5 cDNA (GenBank No.: NM_001285628.1) was subcloned into the pAdTrack-CMV plasmid vector to generate pAdTrackCMV-ELOVL5 vectors. The vector was inserted into an adenoviral vector (pAdEasy-1) to generate adenoviral plasmids in BJ5183 cells. The adenoviral plasmids linearized by PacI (New England Biolabs, Ipswich, MA) were transfected into $293 \mathrm{~A}$ cells to generate the adenovirus pAd-ELOVL5.

\section{Cell Culture}

The GMEC were isolated from peak-lactation Xinong Saanen goats as described previously (Wang et al., 2010; Shi et al., 2014). Details of the cell culture were described recently (Lin et al., 2013; Shi et al., 2013b). Briefly, cells were incubated at $37^{\circ} \mathrm{C}$ in $5 \% \mathrm{CO}_{2}$ and air. Culture medium was composed of Dulbecco's modified Eagle medium/F12 (Hyclone, Beijing, China) containing insulin $(5 \mathrm{mg} / \mathrm{L}$, Sigma-Aldrich, St. Louis, MO), hydrocortisone (5 mg/L, Sigma-Aldrich), penicillin/streptomycin (10k U/L, Harbin Pharmaceutical Group, China), epidermal growth factor (1 mg/L, Sigma-Aldrich), and fetal bovine serum (10\%, Gibco, Waltham, MA). To promote lactogenesis, GMEC were cultured in a lactogenic medium for $24 \mathrm{~h}$ before initial experiments. Lactogenic medium contained the culture medium plus prolactin $(2 \mu \mathrm{g} / \mathrm{mL}$, Sigma-Aldrich). The 293A cells for adenovirus generation were cultured in basal DMEM medium (Gibco) containing 10\% fetal bovine serum.

The GMEC at about $80 \%$ confluence were transfected with adenovirus supernatant (Ad-ELOVL5 or Ad-GFP). The transfected GMEC were collected after $48 \mathrm{~h}$ of culture for lipid extraction, total RNA extraction, and TAG assay.

\section{RNA Interference}

For ELOVL5 mRNA interference, cells cultured in 6-well plates were transfected with $60 \mathrm{n} M$ small interfering RNA (siRNA) using transfection reagent (Lipofectamine RNAiMAX, Thermo Fisher Scientific Inc., Waltham, MA) in lactogenic medium without antibiotic. The transfection was performed according to manufacturer's instructions. Transfected GMEC were collected after $24 \mathrm{~h}$ of initial culture, and then harvested at $48 \mathrm{~h}(24 \mathrm{~h}$ later $)$ for RNA extraction, 
TAG assays, and fatty acid extraction. Sequences of the siRNA oligonucleotides for ELOVL5 (accession: NM_001285628.1; siELOVL5) were designed and synthesized by Jima Biotechnology Co. Ltd. (Shanghai, China). The sequences for the siELOVL5 are described in Supplemental Table S1 (https://doi.org/10.3168/jds .2017-14061). Scrambled siRNA is a functional nontargeting siRNA that is used as a negative control (siNC).

\section{Total RNA Extraction and Quantitative Real-Time PCR}

Total RNA from GMEC was extracted using the RNA Prep Pure Cell Kit (Tiangen Biotech Co. Ltd., Beijing, China) according to the manufacturer's protocol (http://www.tiangen.com/asset/imsupload/ up0203129001467351047.pdf). Genomic DNA contamination was removed using DNase provided with the kit. Synthesis of cDNA was conducted using the PrimeScript RT reagent Kit with gDNA Eraser (Takara Bio Inc., Otsu, Japan) according to the manufacturer's instructions (http://www.takarabiomed.com.cn/Product.aspx $? \mathrm{~m}=20141215102916640154 \# \mathrm{p})$. The quantitative realtime PCR (qPCR) was performed according to the manufacturer's instructions using SYBR Green (SYBR Premix Ex Taq II, Perfect Real Time, Takara Bio Inc.).

Several genes related to TAG synthesis [diacylglycerolacyltransferase1 (DGAT1), DGAT2, perilipin2 (PLIN2)] or SCD1, fatty acid desaturase 1 (FADS1), and FADS2 were selected to evaluate functional outcomes after alteration of ELOVL5 expression. All the qPCR reactions were performed in a Bio-Rad CFX96 (Bio-Rad Laboratories Inc., Hercules, CA) sequence detector, and data were normalized to ubiquitously expressed transcript $(U X T)$, mitochondrial ribosomal protein L39 (MRPL39), and ribosomal protein S9 (RPS9; Bionaz and Loor, 2008). The primer sequences of the genes are described in Supplemental Table S2 (https://doi.org/10.3168/jds.2017-14061).

\section{Fatty Acid Analysis}

After washing 3 times with PBS, treated GMEC were scraped off the culture dish with a $2-\mathrm{mL}$ aliquot of $2.5 \%$ (vol/vol) vitriol:methanol. Total lipid extraction and methylation were performed according to Shi et al. (2013a). The methyl ester of margaric acid (17:0) was used as the internal standard. Methylated lipid samples were analyzed using a GC-MS (Agilent Technologies, Santa Clara, CA) installed with a DB-23 column following a published procedure (Shi et al., 2017b). The relative proportions of fatty acids were determined as the ratio of each fatty acid peak relative to the total fatty acid peaks identified.

\section{Cellular TAG Assays}

The protocol for cellular TAG assay was described previously (Kang et al., 2015; Shi et al., 2015b). Briefly, total cellular TAG was extracted using the GPOTrinder triglyceride assay kit (http://www.applygen .com/a/meixueyushenghuaceding/276.html; Applygen Technologies, Beijing, China). The concentration of TAG was determined according to the manufacturer's instructions on a plate reader (Thermo Fisher Scientific). The concentrations were calculated using the equation obtained from a linear regression of the standard curve, which was established according to the manufacturer's procedure (http://www.applygen.com/ a/meixueyushenghuaceding/276.html). The quantification of total cellular TAG was normalized to the cellular protein concentration. Protein concentration of each well was determined using a BCA protein assay kit (Pierce, Thermo Fisher Scientific) according to the manufacturer's instructions (https://www.thermofisher .com/order/catalog/product/23225).

\section{Statistical Analysis}

Treatments were replicated at least 3 times in culture wells, and the PCR was performed in triplicate. Results are expressed as mean \pm standard error of the mean. Data of qPCR were analyzed using the $2^{-\Delta \Delta \mathrm{Ct}}$ method. Significance of RNA expression of ELOVL5 in mammary tissue across different stages of lactation was determined by 1-way ANOVA. The data for qPCR, TAG content, and fatty acid ratio after altering ELOVL5 expression were determined via Student's $t$-test. Significance was declared at $P<0.05$.

\section{RESULTS}

\section{ELOVL5 Expression in Dairy Goat Mammary Gland Tissue}

As shown in Figure 1, the nonlactating mammary gland had the highest reads per kilobase of exon model per million mapped reads value for ELOVL5, followed by the late-lactation samples $(P<0.05)$. Among the 3 stages, we detected lowest expression of ELOVL5 at peak lactation $(P<0.05)$.

\section{Overexpression of ELOVL5 Altered Genes Related to TAG Synthesis and Fatty Acid Desaturation}

Compared with controls (Ad-GFP), the expression of ELOVL5 after $48 \mathrm{~h}$ increased markedly in cells infected with Ad-ELOVL5 $(P<0.01$, Figure 2A). Compared with the control (Ad-GFP), overexpression of ELOVL5 
significantly upregulated the expression of PLIN2 $(P<$ $0.05)$ and downregulated the expression of DGAT2 $(P$ $<0.05)$, whereas it had no effect on the DGAT1 $(P>$ $0.05)$ mRNA level (Figure 2B).

Regarding the genes related to fatty acid desaturation, compared with control (Ad-GFP), overexpression of ELOVL5 had no significant effect on SCD1 and FADS1 mRNA level. However, it significantly decreased the mRNA level of FADS2 $(P<0.05$; Figure $2 \mathrm{C})$.

\section{Overexpression of ELOVL5 Decreased the Concentration of C16:1}

As shown in Figure 3, overexpression of ELOVL5 significantly decreased the concentration of C16:1n-7 $(P<0.05)$. However, overexpression of ELOVL5 had no effect on the concentration of C16:0, C18:0, C18:1n -7, C18:1n-9, and C18:2.

\section{Knockdown of ELOVL5 Altered Genes Related to TAG Synthesis and Fatty Acid Desaturation}

As shown in Figure 4A, treatment of GMEC with siRNA significantly reduced ELOVL5 mRNA abundance by $92 \%(P<0.01)$. Compared with control (Ad-GFP), knockdown of ELOVL5 downregulated the mRNA level of PLIN2 $(P<0.05$; Figure $4 \mathrm{~B})$. However, no significant changes were observed for the mRNA ex-

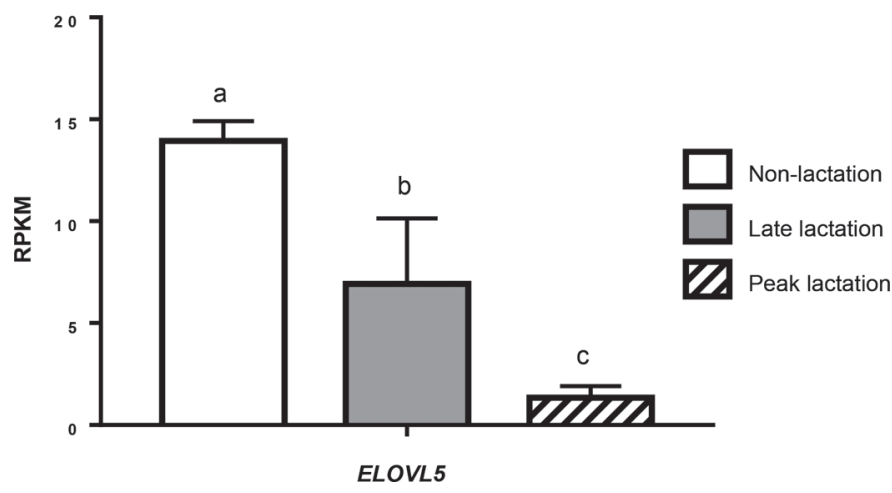

Figure 1. The mRNA abundance of elongation of very long chain fatty acid-like fatty acid elongase $5(E L O V L 5)$ at 3 stages of lactation in goat mammary tissues. The transcriptome data set is from mammary tissue harvested at peak lactation (3 goats, $100 \mathrm{~d}$ postpartum), late lactation (cessation of milking, 3 goats, $310 \mathrm{~d}$ postpartum), and the nonlactating period (3 goats, nonlactation and nonpregnant period) by a veterinarian after slaughter. The DESeq software (http://www huber.embl.de/users/anders/DESeq) was used for abundance analysis of gene expression. Reads per kilobase of exon model per million mapped reads (RPKM) were used for assessing gene expression. Values are means \pm SEM for 3 individual goats. Significance of ELOVL5 expression in mammary tissue across different stages of lactation was determined by 1-way ANOVA. The different letters denote significant $(P<0.05)$ differences.
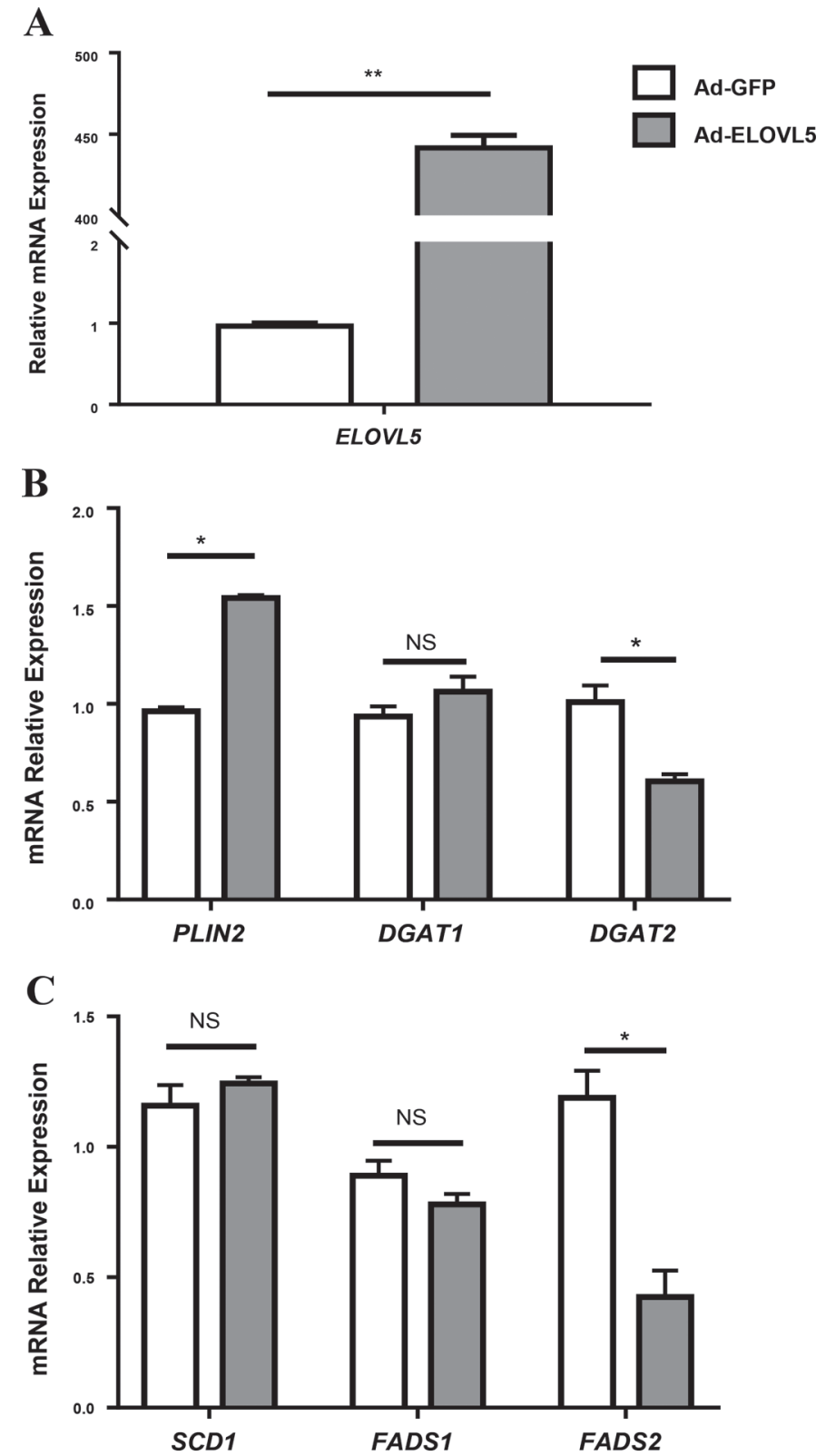

Figure 2. Overexpression of elongation of very long chain fatty acid-like fatty acid elongase 5 (ELOVL5) altered genes related to triacylglycerol synthesis and desaturation. The goat mammary epithelial cells (GMEC) were transfected with recombinant adenovirus expressing ELOVL5 (Ad-ELOVL5) or green fluorescent protein (Ad-GFP) for $48 \mathrm{~h}$ and then collected for mRNA extraction. Panel A: mRNA expression of ELOVL5. Panel B: mRNA expression of genes related to triacylglycerol synthesis (DGAT1, DGAT2, and PLIN2). Panel C: mRNA expression of genes related to desaturation (SCD1, FADS1, and FADS2). Values are means \pm SEM from 3 individual cultures. ${ }^{* *} P<0.01$ compared with control (Ad-GFP). ${ }^{*} P<0.05$ compared with control (Ad-GFP). NS represents no significant change compared with control (Ad-GFP). The data were analyzed via Student's $t$-test. Significance was declared at $P<0.05$. 


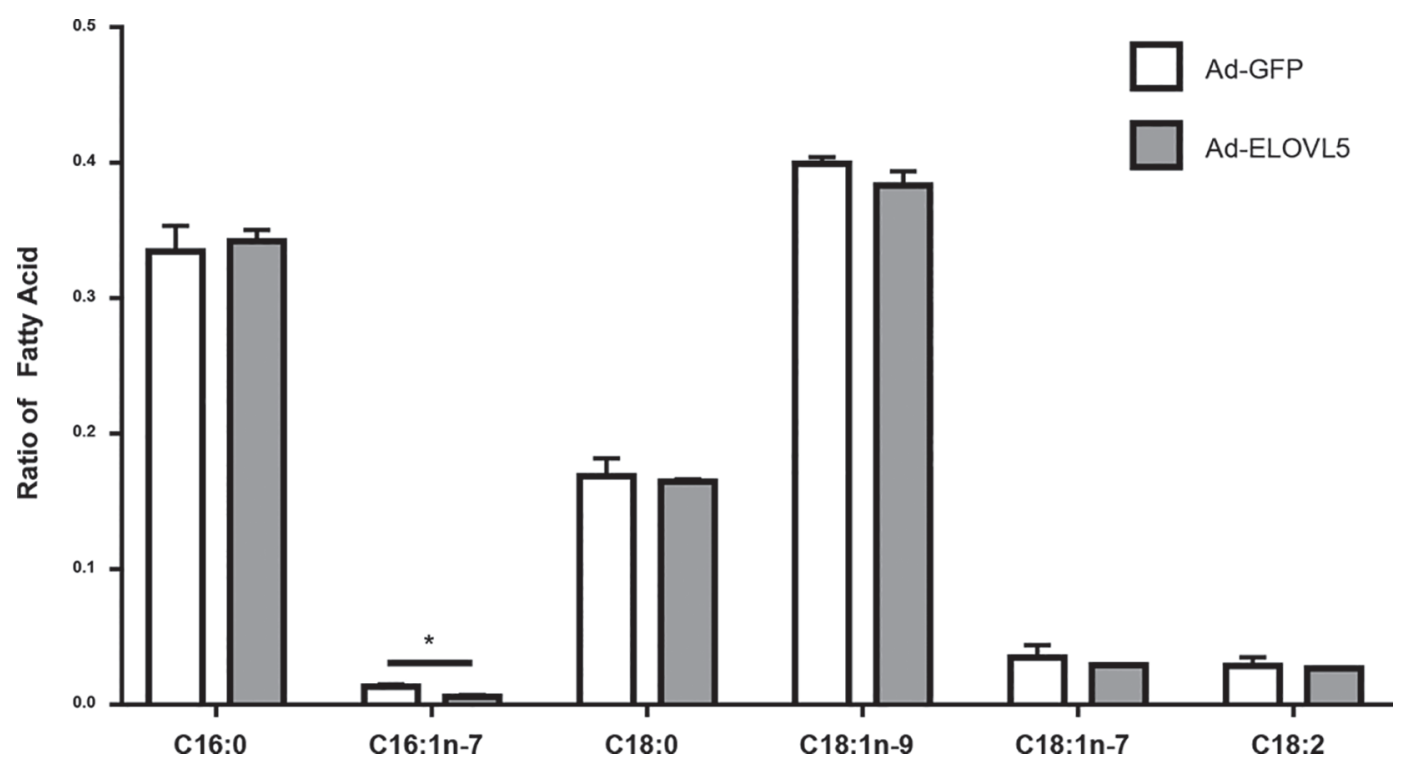

Figure 3. Overexpression of ELOVL5 altered the concentration of C16 to C18 carbon fatty acids in goat mammary epithelial cells (GMEC). The GMEC were transfected with recombinant adenovirus expressing ELOVL5 (Ad-ELOVL5) or green fluorescent protein (Ad-GFP) for $48 \mathrm{~h}$ and then collected for fatty analysis. Data are reported as ratio of total fatty acids. Values are means \pm SEM from 3 individual cultures. The data were analyzed via Student's $t$-test. ${ }^{*} P<0.05$ compared with control (Ad-GFP).

pression level of DGAT1, DGAT2, SCD1, FADS1, and FADS2 in the cells incubated with siELOVL5 (Figures $4 \mathrm{~B}$ and $4 \mathrm{C})$.

\section{Knockdown of ELOVL5 Altered the Concentration of LCFA}

Compared with the control group incubated with siNC, the relative concentration of $\mathrm{C} 18: 1 \mathrm{n}-7$ in GMEC incubated with siELOVL5 decreased significantly $(P<$ 0.05). In contrast, knockdown of ELOVL5 resulted in a marked increase of $\mathrm{C} 16: 1(P<0.05$; Figure 5$)$. No significant changes were observed in the concentration of C16:0, C18:0, C18:1n-7, and C18:2 after knockdown of ELOVL5.

\section{Alteration of ELOVL5 mRNA Expression Had No Effect on Cellular TAG in GMEC}

As shown in Figure $6 \mathrm{~A}$ and $6 \mathrm{~B}$, the concentration of cellular TAG increased did not change significantly $(P$ $>0.05)$ after overexpression or knockdown of ELOVL5 in GMEC.

\section{DISCUSSION}

The LCUFA are bioactive cellular components of membrane phospholipids and can act as direct modulators of gene expression (Mashek and Coleman, 2006).
In humans and rats, desaturases and elongases control the endogenous synthesis of LCUFA (Zhang et al., 2016). A critical role of ELOVL5 in elongating fatty acid substrates ranging from 16 to 20 carbons was reported in humans and rodents (Tripathy et al., 2014). However, whether this enzyme plays the same role in ruminant mammary cells has not been addressed. The present study is novel in that we studied the selective regulation of gene expression and LCFA composition by ELOVL5 in GMEC through altering ELOVL5 expression. Thus, the present findings underscore an important role for ELOVL5 in altering the elongation of 16-carbon to 18-carbon UFA.

The markedly lower expression of ELOVL5 at peak lactation compared with the nonlactating and latelactating period suggests a different role for ELOVL5 during lactation. In the physiological context of milk fat synthesis in the lactating mammary gland, the present data agree with the observation in mouse liver that a consequence of decreased Elovl5 activity is the activation of sterol regulatory element binding protein 1c (SREBP1c) and its target lipogenic genes (Moon et al., 2009). This negative relationship between ELOVL5 and lipogenesis is supported by the high expression of $S R E B P-1 c$ and its target genes in lactating bovine (Bionaz and Loor, 2008) and goat mammary tissue (Shi et al., 2015a). The low expression of ELOVL5 at peak of lactation agrees with the fact that long-chain fatty acids in ruminant milk are mainly taken up from the 
A

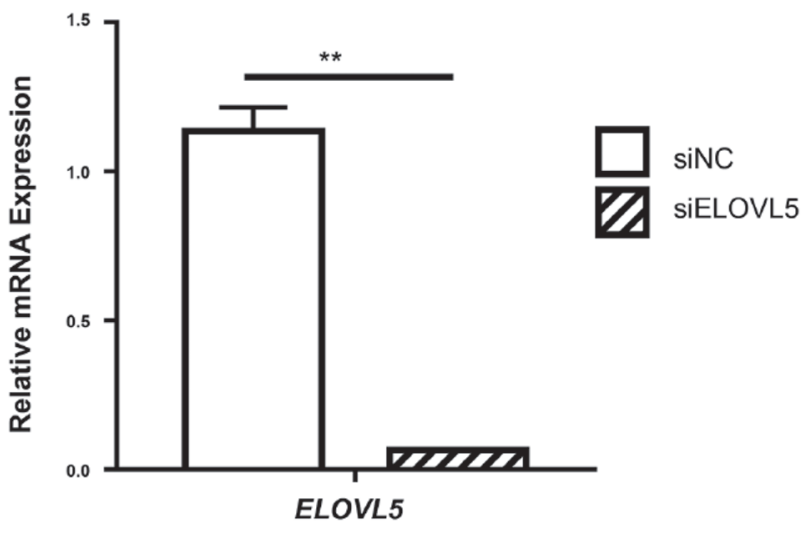

B

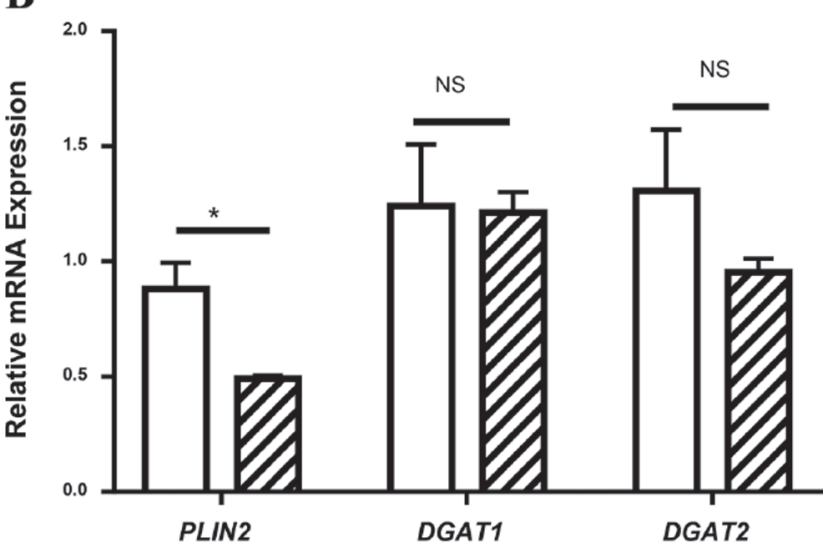

C

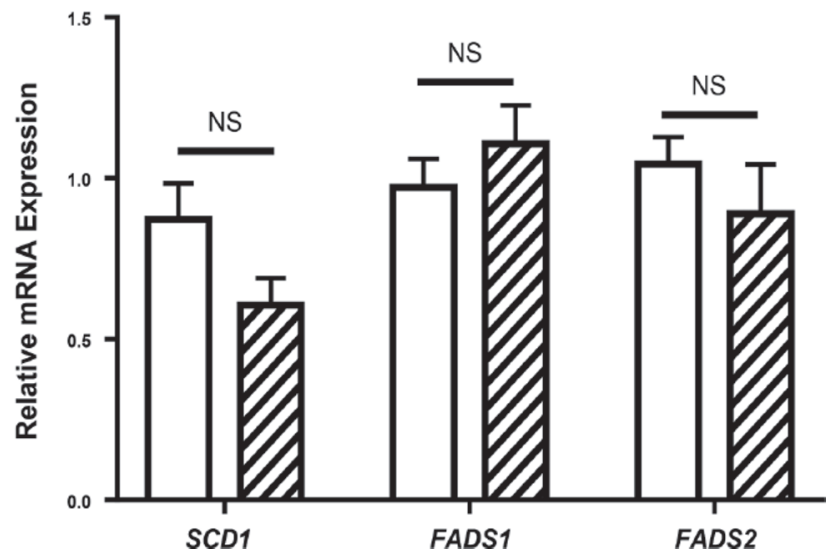

Figure 4. Knockdown of elongation of very long chain fatty acidlike fatty acid elongase 5 (ELOVL5) altered genes related to triacylglycerol synthesis and desaturation. The goat mammary epithelial cells (GMEC) were incubated with small interfering RNA target ELOVL5 (siELOVL5) or negative control (siNC) for $48 \mathrm{~h}$ and then collected for mRNA extraction. Panel A: mRNA expression of ELOVL5. Panel B: mRNA expression of genes related to triacylglycerol synthesis (DGAT1, DGAT2, and PLIN2). Panel C: mRNA expression of genes related to desaturation (SCD1, FADS1, and FADS2). Values are means \pm SEM from 3 individual cultures. The data were analyzed via Student's $t$-test. ${ }^{* *} P<0.01$ compared with control (siNC). ${ }^{*} P<0.05$ compared with control (siNC). NS represents no significant change compared with control (siNC). circulating plasma pool (Harvatine et al., 2009). Together with the fact that ELOVL5 catalyzes the elongation of C18:3n-6 to C20:3n-6 and C18:4n-3 to C20:4n -3 , which in turn negatively affects SREBP-1 activity (Moon et al., 2009), the higher expression of ELOVL5 in dry and late-lactating stages suggest that ELOVL5 may participate in the development of goat mammary tissue through its endogenous products.

The Elovl5 substrates include C16:1n-7 and PUFA such as C18:2n-6 and C18:4n-3 (Zhang et al., 2016). Elongation of fatty acids by Elovl5 is essential for hepatic lipid metabolism in mice, as overexpression of Elovl5 in liver decreases TAG content (Wang et al., 2008) and knockdown of Elovl5 leads to fatty liver and increased lipogenic gene expression (Moon et al., 2009). In the present study, reduction of ELOVL5 expression in GMEC decreased the elongation of C16:1n-7 to C18:1n-7 and overexpression of ELOVL5 decreased the concentration of $\mathrm{C} 16: 1 \mathrm{n}-7$, illustrating a role for ELOVL5 in the elongation of C16:1n-7. This finding agrees with data in insulinoma-1 cells (Wang et al., 2008). The fact that overexpression of ELOVL5 in GMEC had no effect on C18:1n-7 was likely due to limited substrate availability as GMEC cultured in vitro have very low concentrations of $\mathrm{C} 16: 1 \mathrm{n}-7$ relative to C16:0. However, despite the marked change in expression of ELOVL5 due to its overexpression, only modest changes in cellular LCUFA composition were detected. Such responses could be partly explained by the lack of exogenous availability of 16:1 for the enzyme reaction and also lack of an increase in ELOVL5 protein. The lack of a goat-specific antibody for ELOVL5 clearly is a limiting factor in the present study. Despite being unable to measure ELOVL5 protein, the present data suggest that the alteration in 16-carbon to 18-carbon UFA composition was a primary result of changes in ELOVL5 mRNA.

The desaturase and elongase work in concert during the biosynthesis of LCUFA (Wang et al., 2006). The lack of effect of ELOVL5 expression on SCD1 in the present study is consistent with the observation in mice with a knockdown of Elovl5. The lack of change in the concentration of C18:1n-9 agreed with the lack of change of SCD1 expression when ELOVL5 was knocked down or upregulated. The finding that in cells overexpressing ELOVL5, expression of FADS2 was downregulated agrees with the data in mouse liver. The downregulation of FADS2 may have been caused by the alteration of fatty acids (such as $\mathrm{C} 16: 1$ or $\mathrm{C} 18: 1 \mathrm{n}-7$ ), which are natural agonists for nuclear transcription factors [e.g., peroxisome proliferator activated receptor (PPAR) or SREBP1]. This idea is supported by data from mice showing that hepatic Elovl5 activity affects multiple 


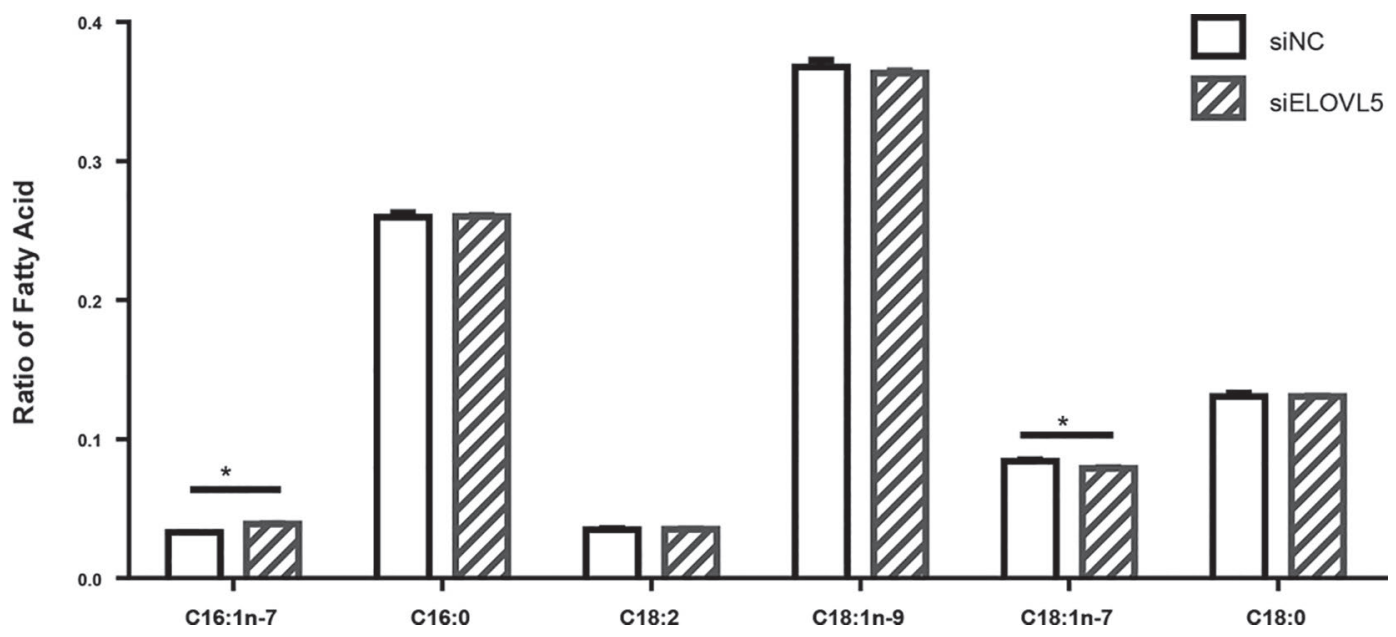

Figure 5. Knockdown of ELOVL5 altered the concentration of C16 to C18 carbon fatty acids in goat mammary epithelial cells (GMEC). The GMEC were incubated with small interfering RNA target ELOVL5 (siELOVL5) or negative control (siNC) for $48 \mathrm{~h}$ and then collected for fatty analysis. Data are reported as ratio of total fatty acids. Values are means \pm SEM from 3 individual cultures. The data were analyzed via Student's $t$-test. ${ }^{*} P<0.05$ compared with control (siNC).

pathways controlling lipid and carbohydrate composition (Wang et al., 2008). The products of ELOVL5 can serve to activate various signaling pathways related to lipid and carbohydrate metabolism (Tripathy et al., 2010, 2014). The lack of change for FADS1 after overexpression or knockdown of ELOVL5 indicated a potential lack of interaction between the 2 genes in vitro. Collectively, the evidence suggests ELOVL5 may play a role in the cellular homeostasis via controlling cellular concentrations of LCUFA (Green et al., 2010), which serve as cell-signaling molecules during lactation.

In ruminants, the enzymes involved in synthesis of TAG including DGAT1 and DGAT2 are upregulated during lactation (Bionaz and Loor, 2008). The decrease in DGAT2 after overexpression of ELOVL5 is consistent with data in mice demonstrating that Elovl5 activity resulted in lower hepatic TAG content (Tripathy et al., 2014). However, overexpression or knockdown of ELOVL5 in GMEC did not alter the content of TAG. The lack of change in TAG might have been due to insufficient substrate in vitro for GMEC. The lack of change in DGAT1 after altering ELOVL5 expression confirmed our previous hypothesis that DGAT1 protein is prone to respond to the availability of endogenous fatty acids for TAG synthesis (Shi et al., 2017a).

The PLIN2 protein is essential for the formation and secretion of lipid droplets during lactation (Shi et al., 2015b) and is a target of PPAR (Kang et al., 2015; Shi et al., 2017b). In the present study, PLIN2 expression had a similar pattern as ELOVL5. This result did not agree with the lack of change in TAG and the fact that Elovl5 activity is associated with a decrease in liver TAG content in mice (Tripathy et al., 2014). However, the response is consistent with evidence that Elovl5 could affect the PPAR or SREBP1 pathway via changes in the concentration of its fatty acid products (Tripathy et al., 2014). It is also worth noting that PLIN2 expression was significantly upregulated when concentration of C16:1 decreased, and was downregulated when concentration of C16:1 increased. This suggests that C16:1 might be the key product or substrate of ELOVL5 that can signal through the PPAR pathway. This idea is supported by data showing that ELOVL5 functions to keep cellular concentrations of $\mathrm{C} 16: 1 \mathrm{n}-7$ low, thereby preventing its accumulation and any potential downstream signaling event (Green et al., 2010). The potential mechanistic role of $\mathrm{C} 16: 1 \mathrm{n}$ -7 in ruminant mammary cells is unclear; thus, more experiments appear warranted. Collectively, the data from the present study suggest that ELOVL5 plays an important regulatory role in controlling LCUFA elongation in goat mammary cells.

\section{CONCLUSIONS}

The upregulation of ELOVL5 decreased the concentration of C16:1n-7, whereas knockdown decreased the concentration of $\mathrm{C} 18: 1 \mathrm{n}-7$ and increased that of $\mathrm{C} 16$ : 1n-7. Activation of ELOVL5 altered the expression of genes associated with fatty acid desaturation and TAG synthesis, and the accumulation of $\mathrm{C} 16: 1 \mathrm{n}-7$ seems to be involved. In conclusion, at least in vitro, these data demonstrate a direct role for ELOVL5 in modulating LCUFA synthesis in GMEC. 

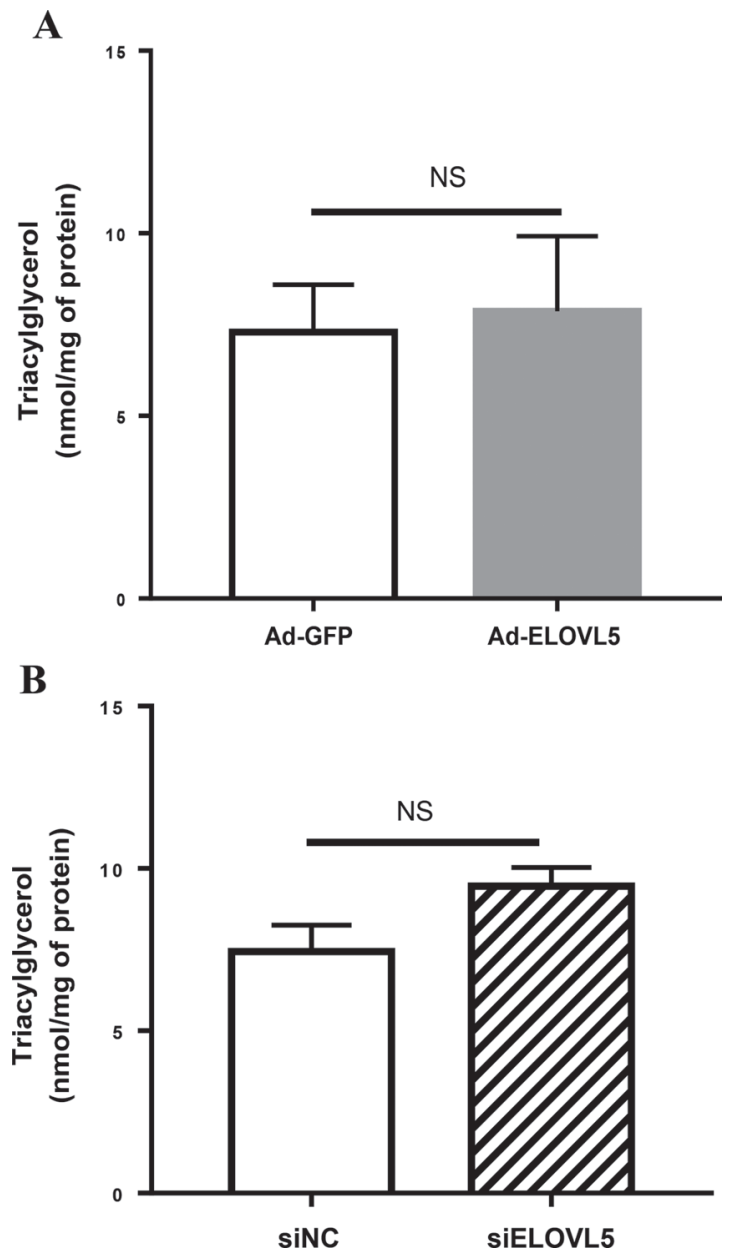

Figure 6. Elongation of very long chain fatty acid-like fatty acid elongase 5 (ELOVL5) did not significantly alter the accumulation of cellular triacylglycerol. The goat mammary epithelial cells (GMEC) were transfected with recombinant adenovirus expressing ELOVL5 (Ad-ELOVL5) or green fluorescent protein (Ad-GFP) or incubated with small interfering RNA target ELOVL5 (siELOVL5) or negative control (siNC), and collected at $48 \mathrm{~h}$ for cellular triacylglycerol analysis. Values are means \pm SEM from 3 individual cultures. The data were analyzed via Student's $t$-test. No significant change compared with control (Ad-ELOVL5 vs. Ad-GFP or siELOVL5 vs. siNC) is denoted by NS.

\section{ACKNOWLEDGMENTS}

This research was supported by National Natural Science Foundation of China (31702090 and 31460585, Beijing, China).

\section{REFERENCES}

Bionaz, M., and J. Loor. 2008. Gene networks driving bovine milk fat synthesis during the lactation cycle. BMC Genomics 9:366.

Cecchinato, A., G. Bittante, C. Ribeca, A. Maurmayr, M. Penasa, M. De Marchi, N. P. P. Macciotta, M. Mele, P. Secchiari, and G. Pagnacco. 2012. Effects of beta-lactoglobulin, stearoyl-coenzyme
A desaturase 1, and sterol regulatory element binding protein gene allelic variants on milk production, composition, acidity, and coagulation properties of Brown Swiss cows. J. Dairy Sci. 95:450-454.

Dong, Y., M. Xie, Y. Jiang, N. Q. Xiao, X. Y. Du, W. G. Zhang, G. Tosser-Klopp, J. H. Wang, S. Yang, J. Liang, W. B. Chen, J. Chen, P. Zeng, Y. Hou, C. Bian, S. K. Pan, Y. X. Li, X. Liu, W. L. Wang, B. Servin, B. Sayre, B. Zhu, D. Sweeney, R. Moore, W. H. Nie, Y. Y. Shen, R. P. Zhao, G. J. Zhang, J. Q. Li, T. Faraut, J. Womack, Y. P. Zhang, J. Kijas, N. Cockett, X. Xu, S. H. Zhao, J. Wang, and W. Wang. 2013. Sequencing and automated wholegenome optical mapping of the genome of a domestic goat (Capra hircus). Nat. Biotechnol. 31:135-141.

Green, C. D., C. G. Ozguden-Akkoc, Y. Wang, D. B. Jump, and L. K. Olson. 2010. Role of fatty acid elongases in determination of de novo synthesized monounsaturated fatty acid species. J. Lipid Res. 51:1871-1877.

Han, S., E. A. Schroeder, C. G. Silva-Garcia, K. Hebestreit, W. B. Mair, and A. Brunet. 2017. Mono-unsaturated fatty acids link H3K4me3 modifiers to C. elegans lifespan. Nature 544:185-190.

Harvatine, K. J., Y. R. Boisclair, and D. E. Bauman. 2009. Recent advances in the regulation of milk fat synthesis. Animal 3:40-54.

Kang, Y., S. Hengbo, L. Jun, L. Jun, Z. Wangsheng, T. Huibin, and S. Huaiping. 2015. PPARG modulated lipid accumulation in dairy GMEC via regulation of ADRP gene. J. Cell. Biochem. 116:192201

Lee, J. M., H. Lee, S. Kang, and W. J. Park. 2016. Fatty acid desaturases, polyunsaturated fatty acid regulation, and biotechnological advances. Nutrients 8. https://doi.org/10.3390/nu8010023.

Lin, X., J. Luo, L. Zhang, W. Wang, H. Shi, and J. Zhu. 2013. MiR27a suppresses triglyceride accumulation and affects gene mRNA expression associated with fat metabolism in dairy goat mammary gland epithelial cells. Gene 521:15-23.

Mashek, D. G., and R. A. Coleman. 2006. Cellular fatty acid uptake: The contribution of metabolism. Curr. Opin. Lipidol. 17:274-278.

Moon, Y.-A., R. E. Hammer, and J. D. Horton. 2009. Deletion of ELOVL5 leads to fatty liver through activation of SREBP-1c in mice. J. Lipid Res. 50:412-423.

Shi, H., H. Shi, J. Luo, W. Wang, A. B. Haile, H. Xu, and J. Li. 2014. Establishment and characterization of a dairy goat mammary epithelial cell line with human telomerase (hT-MECs). Anim. Sci. J. $85: 735-743$.

Shi, H., W. Zhao, C. Zhang, K. Shahzad, J. Luo, and J. J. Loor. 2016. Transcriptome-wide analysis reveals the role of PPARG controlling the lipid metabolism in goat mammary epithelial cells. PPAR Res. 2016:9195680.

Shi, H., J. Zhu, J. Luo, W. Cao, H. Shi, D. Yao, J. Li, Y. Sun, H. $\mathrm{Xu}, \mathrm{K}$. Yu, and J. Loor. 2015a. Genes regulating lipid and protein metabolism are highly expressed in mammary gland of lactating dairy goats. Funct. Integr. Genomics 15:309-321.

Shi, H. B., J. Luo, D. W. Yao, J. J. Zhu, H. F. Xu, H. P. Shi, and J. J. Loor. 2013a. Peroxisome proliferator-activated receptor-Y stimulates the synthesis of monounsaturated fatty acids in dairy goat mammary epithelial cells via the control of stearoyl-coenzyme A desaturase. J. Dairy Sci. 96:7844-7853.

Shi, H. B., J. Luo, J. J. Zhu, J. Li, Y. T. Sun, X. Z. Lin, L. P. Zhang, D. W. Yao, and H. P. Shi. 2013b. PPAR $\gamma$ regulates genes involved in triacylglycerol synthesis and secretion in mammary gland epithelial cells of dairy goats. PPAR Res. 2013:310948.

Shi, H. B., M. Wu, J. J. Zhu, C. H. Zhang, D. W. Yao, J. Luo, and J. J. Loor. 2017a. Fatty acid elongase 6 plays a role in the synthesis of long-chain fatty acids in goat mammary epithelial cells. J. Dairy Sci. 100:4987-4995.

Shi, H. B., K. Yu, J. Luo, J. Li, H. B. Tian, J. J. Zhu, Y. T. Sun, D. W. Yao, H. F. Xu, H. P. Shi, and J. J. Loor. 2015b. Adipocyte differentiation-related protein promotes lipid accumulation in goat mammary epithelial cells. J. Dairy Sci. 98:6954-6964.

Shi, H. B., C. H. Zhang, W. Zhao, J. Luo, and J. J. Loor. 2017b. Peroxisome proliferator-activated receptor delta facilitates lipid secretion and catabolism of fatty acids in dairy goat mammary epithelial cells. J. Dairy Sci. 100:797-806. 
Shingfield, K. J., Y. Chilliard, V. Toivonen, P. Kairenius, and D. I. Givens. 2008. Trans fatty acids and bioactive lipids in ruminant milk. Adv. Exp. Med. Biol. 606:3-65.

Tripathy, S., K. A. Lytle, R. D. Stevens, J. R. Bain, C. B. Newgard, A. S. Greenberg, L. S. Huang, and D. B. Jump. 2014. Fatty acid elongase-5 (Elovl5) regulates hepatic triglyceride catabolism in obese C57BL/6J mice. J. Lipid Res. 55:1448-1464.

Tripathy, S., M. Torres-Gonzalez, and D. B. Jump. 2010. Elevated hepatic fatty acid elongase- 5 activity corrects dietary fat-induced hyperglycemia in obese C57BL/6J mice. J. Lipid Res. 51:2642-2654.

Wang, Y., D. Botolin, J. H. Xu, B. Christian, E. Mitchell, B. Jayaprakasam, M. G. Nair, J. M. Peters, J. V. Busik, L. K. Olson, and D. B. Jump. 2006. Regulation of hepatic fatty acid elongase and desaturase expression in diabetes and obesity. J. Lipid Res. $47: 2028-2041$
Wang, Y., M. Torres-Gonzalez, S. Tripathy, D. Botolin, B. Christian, and D. B. Jump. 2008. Elevated hepatic fatty acid elongase-5 activity affects multiple pathways controlling hepatic lipid and carbohydrate composition. J. Lipid Res. 49:1538-1552.

Wang, Z., J. Luo, W. Wang, W. Zhao, and X. Lin. 2010. Characterization and culture of isolated primary dairy goat mammary gland epithelial cells. Chin. J. Biotechnol. 26:1123-1127.

Yao, D., J. Luo, Q. He, H. Shi, J. Li, H. Wang, H. Xu, Z. Chen, Y. Yi, and J. J. Loor. 2017. SCD1 alters long-chain fatty acid (LCFA) composition and its expression is directly regulated by SREBP-1 and PPARgamma 1 in dairy goat mammary cells. J. Cell. Physiol. 232:635-649.

Zhang, J. Y., K. S. D. Kothapalli, and J. T. Brenna. 2016. Desaturase and elongase limiting endogenous long chain polyunsaturated fatty acid biosynthesis. Curr. Opin. Clin. Nutr. Metab. Care 19:103-110. 